\title{
Endoscopic nasobiliary tube drain (ENBTD) prior to laparoscopic cholecystectomy in acute \pm complicated cholecystitis: Influence on the rate of complications
}

\author{
Ayman M.Soliman, MD; Ahmed M. Awad, MD; \\ Yasser A. ElReheem, MD; Usama El Attar, FRCS \\ Department of General Surgery, Ain Shams University, Cairo, Egypt.
}

\begin{abstract}
Background and aim: It is not known to what extent surgical outcome of laparoscopic cholecystectomy for acute or complicated cholecystitis differ from those for the chronic form, making it questionable whether urgent laparoscopic cholecystectomy is the best approach even in severe acute cases. Also, risk factors may predispose to bile duct injury or postoperative bile leakage associated with laparoscopic cholecystectomy $(L C)$ including the presence of an accessory hepatic duct, the anomalous cystic duct confluence, and duct of Luschka. A method to prevent the bile duct injury is a preoperative placement of an endoscopic nasobiliary tube drain (ENBTD). The aim of this review was to evaluate the efficacy of ENBTD assisted LC regarding prevention of intraoperative and postoperative bile duct injury or leakage.

Methods: From April 2009 to June 2011, a total of 96 consecutive ENBTD assisted LCs for acute cholecystitis \pm complicated cholecystitis performed in our institutions were reviewed during this period.

Results: Anomalous cystic duct confluence was detected in 3 cases and an accessory hepatic duct was detected in 2 cases. These anomalies were risk factors for bile duct injury in our series. However, there was no significant difference in the length of surgery or conversion rate to laparotomy, but significant decrease in the frequency of bile duct injury and leakage compared to the standard $L C$.

Conclusion: Bile duct anomalies were seen in $6.72 \%$ of LC cases. Placement of an ENBTD tube prior to LC in acute cholecystitis \pm empyema or gangerenous cholecystitis had successfully decreased the incidence of complications.

Key words: Laparoscopic cholecystectomy, endoscopic nasobiliary tube drain, bile duct injury.
\end{abstract}

\section{Introduction:}

Laparoscopic management of acute cholecystitis is a logical progression from elective laparoscopic cholecystectomy, but questions remain about its safety, cost effectiveness, and success rate. ${ }^{1}$ Although laparoscopic cholecystectomy is the procedure of choice in chronic cholecystitis, its use in acute cholecystitis may be associated with higher costs and complication rates. Also, It is not known which patients with acute cholecystitis are likely to require conversion to open cholecystectomy based on preoperative data or if a cooling-off period with medical therapy can diminish inflammation and increase the chance of successful laparoscopic cholecystectomy.,2,3

Intraoperative bile duct injury and/ or postoperative bile leakage have been considered among the most serious complications of laparoscopic cholecystectomy (LC), resulting in chronic 
morbidity and even mortality. The incidence has been reported to be $0.3-3.4 \%$ by several authors. ${ }^{4}$ The risk factors predisposing to bile duct injury or postoperative bile leakage include the presence of an accessory hepatic duct, the anomalous cystic duct confluence, and duct of Luschka. ${ }^{3,4}$ Therefore, it is crucial to be aware of these anomalies prior to surgery to help prevent bile duct injury. ${ }^{5}$ Although there are many series that show the safety of laparoscopic cholecystectomy, the true incidence of bile duct injury is undoubtedly, most bile duct injuries occur in cases of acute cholecystitis and are not prevented by intraoperative cholangiography. ${ }^{6}$ A method to prevent bile duct injury is preoperative placement of an endoscopic nasobiliary tube drain (ENBTD). ENBTD assisted LC; this technique was first proposed by Uchiyama et al. in 2006 and was advocated by several others especially for situations prone to bile duct injury, such as acute cholecystitis, anticipated severe inflammation, and the presence of bile duct anomalies. ${ }^{7}$ We have performed ENBTD assisted LC for all cases in which acute cholecystitis \pm bile duct anomalies as recognized preoperatively.

In this study we evaluate the surgical outcomes of laparoscopic cholecystectomy for cholecystitis \pm acute complicated cholecystitis \pm presence of bile duct anomalies. Also, to highlight the role and the efficacy of ENBTD assisted LC with regard to prevention of intraoperative and postoperative bile duct injury or leakage.

\section{Patients and methods:}

This study was conducted over ninety six patients presented with acute calcular cholecystitis in Ain Shams University Hospitals (Egypt) and in Obeid Hospital, Armed Forces Hospital King Abdulaziz Naval Base and Soliman Fakeeh Hospital (Saudi Arabia) during the period between April 2009 to June 2011. Patients with acalculous cholecystitis were excluded. For patients with cholecystitis that was associated with organ or system dysfunction, a percutaneous cholecystostomy tube was inserted as an alternative to operating on high-risk patients and thereby converting their operation to an elective procedure, those patients were also excluded. For all cases (acute \pm complicated cholecystitis) bile duct anatomy was evaluated by (ERCP) with placement of an ENBTD. Figure(1,2) Endoscopic nasobiliary tube (ENBTD) placement technique was as described by Soehendra 8 : Nasobiliary catheter was applicated over the guide wire passing through the biopsy channel of the endoscope and its tip was positioned above the bifurcation of the CBD without concomitant sphincterotomy (except if done during therapeutic ERCP). Guide wire was then withdrawn followed by the endoscope. After ensuring a free flow of bile from the external end of ENBD catheter, the catheter was rerouted through the nose by a rail-road technique using a nasogastric tube which passed transnasally; and finally, the endoscopist passed his fingers into the patient's mouth to retrieve the transfer tube from the oropharynx to allow the nasobiliary tube to be transferred from mouth to nose. A cholangiogram was repeated through the ENBD catheter to adequately visualize the biliary system and to confirm its position. Then ENBD catheter, while in position, was irrigated using $20 \mathrm{ml}-60 \mathrm{ml}$ of sterile normal saline.

The ENBTD types are shown in table 1. In this study we used $(5,7 \mathrm{~F}$ straight or pigtailed tip ENTD tube, Olympus MS, long $\alpha, 4,9$ side openings) Figure(3) Each case was carefully watched for post ENBTD pancreatitis. Laparoscopic cholecystectomy was performed using the standard 3- or 4-port technique one day after ENBTD application with/without intraoperative cholangiography (IOC).

As previously described, AC was defined when the patient had 2 or more of the following clinical and operative findings. The clinical factors consisted of: fever with a body temperature higher than $37.5^{\circ} \mathrm{C}$, leukocytosis and right upper abdominal pain with tenderness continuous for more than 48-hour duration despite medical treatment. Ultrasound findings included gallbladder wall thickness (edema) of greater than $4 \mathrm{~mm}$ 


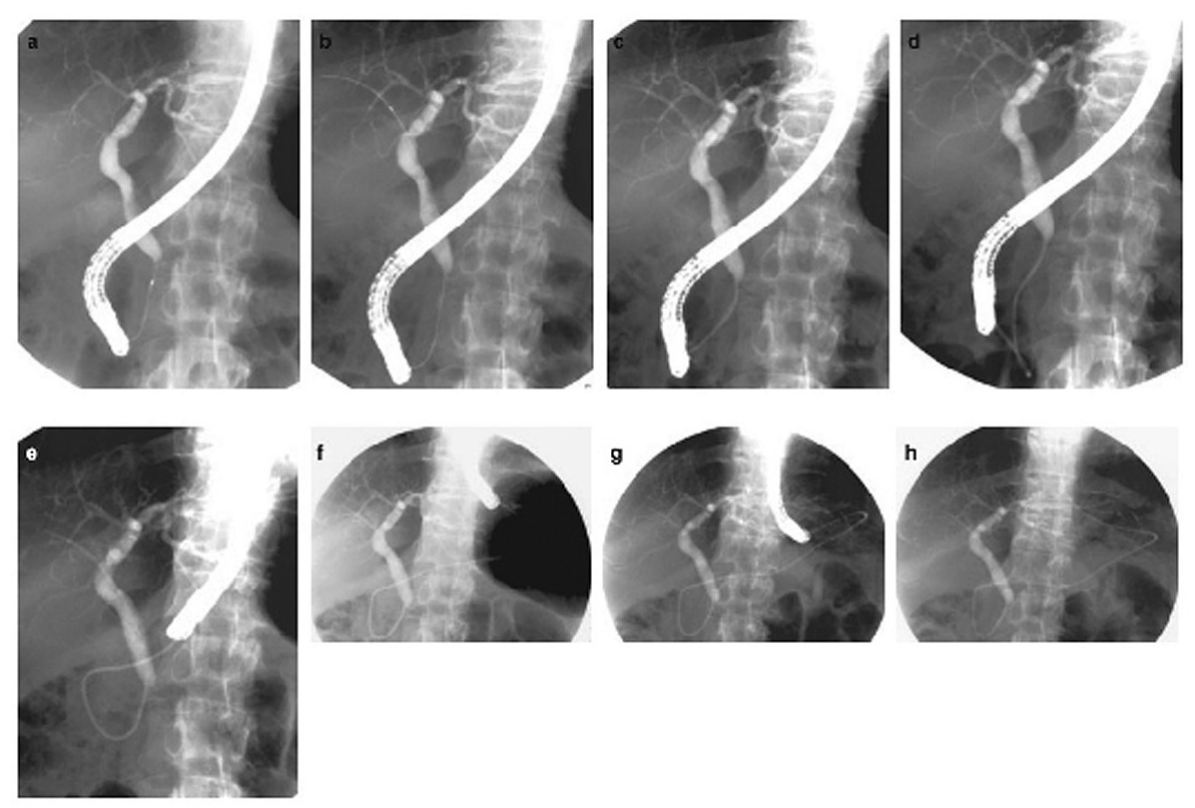

Figure (1): Technique of placement of endoscopic nasobiliary drainage (ENBD).

(a) Endoscopic retrograde cholangiopancreatography showed lower bile duct stricture.

(b) Deep cannulation with guidewire.

(c) Long a-type ENBD tube was indwelling right intrahepatic duct.

(d) The tube was advanced approximately $5 \mathrm{~cm}$ or more.

(e) The scope was withdrawn into the stomach.

(f) The tube was adequately bent at the fornix of the stomach.

(g) Air in the stomach was aspirated.

(h) Adequate observation of the intragastric tube was possible.
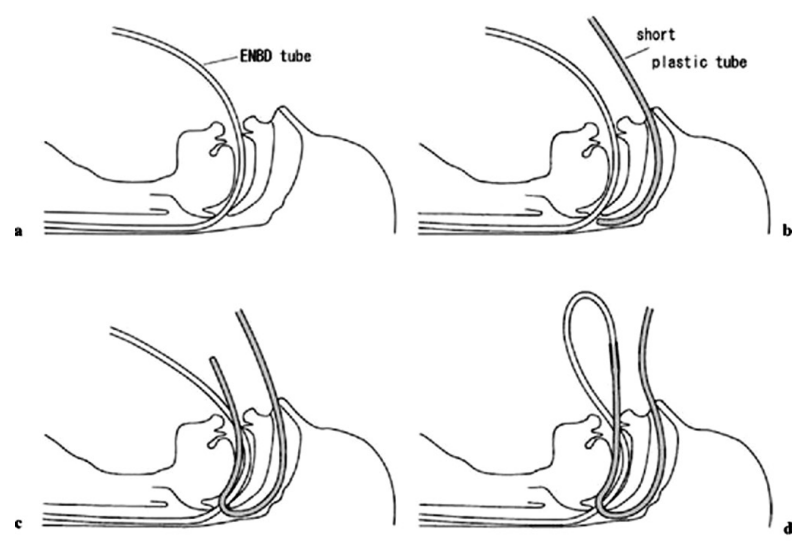

Figure (2): Technique of conversion of the oral exit of the ENBTD tube to nasal exit a) An endoscopic catheter was cannulated into the bile duct. b) A guide catheter was passed through the nasal opening to the
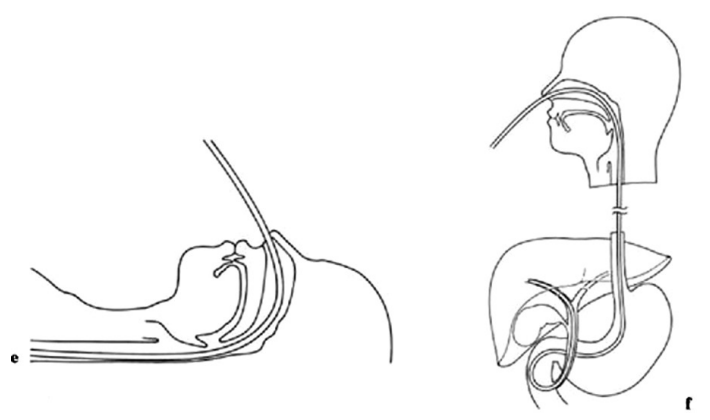
pharynx. c) The catheter was withdrawn through the mouth. d) The ENBTD tube was fixed to the guide catheter. e) The guide catheter was withdrawn through the nose. f) The ENBTD tube was fixed to keep it in place. 
Table (1): Commercially available ENBD tubes.

\begin{tabular}{|l|c|c|c|c|}
\hline Products & Diameter (Fr) & Shape of tip & Shape of distortion & Side hole \\
\hline Olympus MS & 5,7 & $\mathrm{~S}, \mathrm{P}$ & $\alpha, 1-\alpha, \mathrm{R} 1-\alpha$ & $\mathrm{P} 9, \mathrm{~S} 4$ \\
\hline Boston SJ & $5,6,7.5,8.5$ & $\mathrm{P}$ & $1-\alpha, \mathrm{R} 1-\alpha$, straight & 7 \\
\hline Cliny & 7 & $\mathrm{~S}, \mathrm{P}$ & $\alpha, 1-\alpha, \mathrm{R} 1-\alpha$, straight & $\mathrm{P} 6, \mathrm{~S} 4$ \\
\hline Hanako & $5,6,7.2,8$ & $\mathrm{~S}, \mathrm{P}$ & Straight & $\mathrm{P} 4, \mathrm{~S} 4$ \\
\hline Wison-Cook & $5,6,7,10$ & $\mathrm{P}$ & $1-\alpha$ & 5 \\
\hline Liguory & $5,6,7$ & $\mathrm{P}$ & $1-\alpha$ & 9 \\
\hline Nagaraja & $5,6,7$ & $\mathrm{~S}$ & $\mathrm{R} 1-\alpha$ & 5 \\
\hline Leung & 5,6 & $\mathrm{~A}$ & & 4 and 7 \\
\hline
\end{tabular}

$A$, angled; ENBD, endoscopic nasobiliary drainage; $P$, pigtailed; $S$, straight.

1 - $\alpha$, long $a$; R1- $\alpha$, reversed-long $\alpha$.
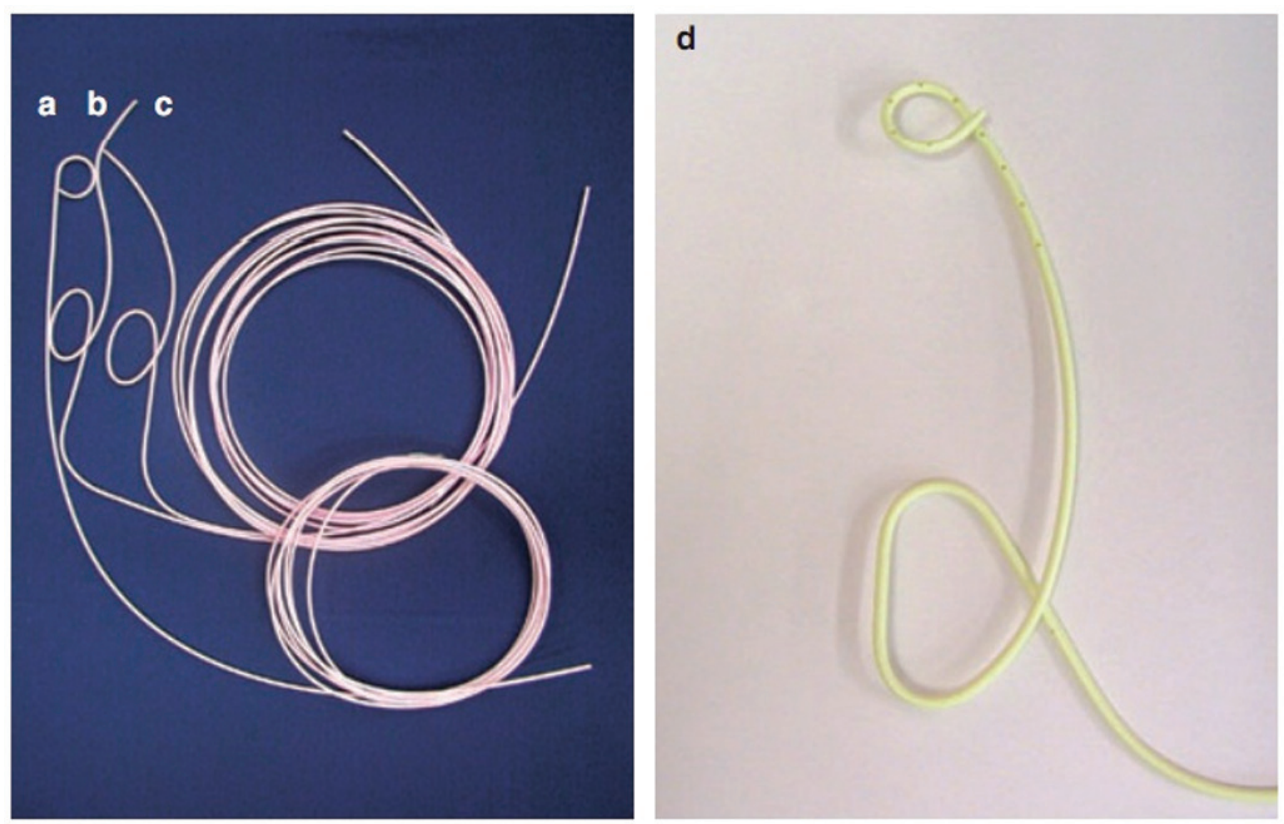

Figure (3): Endoscopic nasobiliary drainage tubes. (a) Pig-tailed tip without distortion, (b) Straight tip with contra $\alpha$-distortion, (c) Straight tip with $\alpha$-distortion, (d) pig-tailed tip with $\alpha$-distortion ( $a-c$, Hanako; d, Olympus).

by Abdominal US and pericholecystic collection. The operative findings include severe adhesion to an adjacent organ, distortion of the biliary anatomy, and gross inflammation of the gallbladder serosa.

Complicated cholecystitis is considered if hydrops, empyema, pericholecystic abscess, or gangrene developed. Figure(4) 


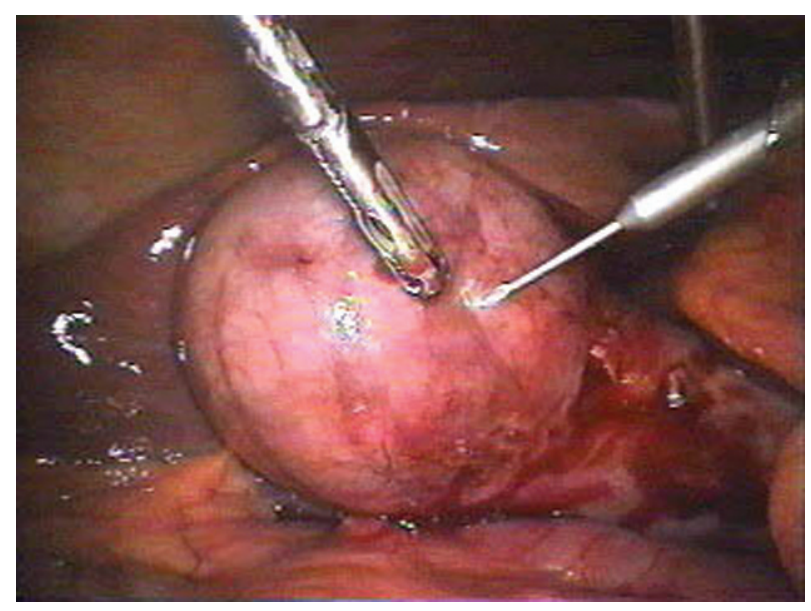

Figure (4): A case of acute cholecystitis with thickened \& inflammed gallbladder wall, with severe adhesion to an adjacent organ.

The data were collected from all consecutive ENBTD assisted LC cases which were performed in our institutions from January 2009 to June 2011 for each patient. Patients were analyzed for sixteen variables: demographics (age, sex), preexisting comorbidities (diabetes mellitus, coronary artery disease, hyperlipidemia), preoperative signs and symptoms (right upper quadrant abdominal pain, nausea, fever); initial laboratory values (leukocytic count, asparate aminotransferase [AST], alanine aminotransferase [ALT], albumin, alkaline phosphatase [ALP], bilirubin, amylase, lipase), and preoperative imaging (ultrasonography). In the basic analysis, the mean and standard deviation for continuous variables and the frequency and proportion for categorical variables were calculated on the entire data set. Univariate analysis for the histological diagnosis, the dependent variable in this study, was performed with Chi-square analysis. The $\mathrm{P}$ value, odds ratio (OR), and 95\% confidence interval (CI) were determined for each variable. The variables with a $P$ value of 0.1 or less were selected for further multivariate analysis.

\section{Results:}

The study was carried on 36 men (37.5\%) and 60 women $(62.5 \%)$, with a mean (SD) age of 57.9 years (range, 23-70 years). The diagnosis of the 96 patients who did LC included AC ( $\mathrm{n}=84$ [87.5\%]), Complicated AC developed in $(n=12$ [12.5\%]) patients. Complicated AC included empyema $(\mathrm{n}=4)$, hydrops $(\mathrm{n}=2)$, gangrenous AC $(\mathrm{n}=1)$, perforation $(n=2)$ and pericholecystic abscess $(n=3)$. The preoperative characteristics of the patients according to the diagnosis are summarized in Table(2).

Results from univariate and multivariate analysis showed that 8 variables were associated with an increased risk of developing AC. Table(3) listed the candidate predictors in the final logistic regression model with their odds ratios (ORs) and corresponding adjusted $\mathrm{P}$ values. American Society of Anesthesiologists score, presence of respiratory disease or chronic liver disease, and history of a previous upper abdominal operation were not significant after multivariate analysis. 
Table (2): Preoperative characteristics of the patients .

\begin{tabular}{|l|c|c|}
\hline Characteristics & No of Patients (96) & P - value \\
\hline Age (years) & $42 \pm 18$ & 0.001 \\
\hline Male/female & $36 / 60$ & 0.001 \\
\hline Total hospital stay (days) & $2.0 \pm 2.7$ & 0.0001 \\
\hline Operative time (minutes) & $44.5 \pm 19.6$ & $<0.001$ \\
\hline Patient Diagnosis: & $84(87.5 \%)$ & \\
- Acute cholecystitis (AC) & $4(4.16 \%)$ & 0.001 \\
- Empema of gall bladder & $2(2.08 \%)$ & 0.0001 \\
- Hydrops of gall bladder & $1(1.04 \%)$ & 0.0001 \\
- Gangernous gall bladder & $2(2.08 \%)$ & 0.00001 \\
- Perforation of gall bladder & $3(3.12 \%)$ & 0.0001 \\
\hline
\end{tabular}

A total of 96 consecutive ENBTD assisted LCs were performed during the study period (male/female 36:60, median age $42 \pm 18$ years). To preoperatively assess the biliary tract, all patients underwent direct cholangiography (ERCP) which discoverd 5 cases of bile duct anomalies. In total, we performed ENBTD assisted LC for all cases. In terms of post ENBTD complication; we experienced a single case of post ENTD pancreatitis in the case of cholecystitis and cholelithiasis. This event was conservatively managed with success and ENBTD assisted LC followed without bile duct injury 3 months later.

Table (3): Prediction for the development of acute cholecystitis.

\begin{tabular}{|l|c|c|c|}
\hline Variables & Cut off & Odds ratio $(95 \% \mathrm{CI})$ & P-value \\
\hline Age, mean (SD). years & $>51$ years & $3.5(1.07-11.40)$ & $<.01$ \\
\hline Sex & & & $<.01$ \\
- Male & $\begin{array}{l}36(37.5 \%) \\
- \text { Female }\end{array}$ & & $<.01$ \\
\hline White blood cell count & $>15 \mathrm{~K} / \mathrm{cmm}$ & $4.38(1.75-10.97)$ & 0.0016 \\
\hline Diabetes mellitus & $12(12.5 \%)$ & $2.84(1.26-6.40)$ & 0.0012 \\
\hline Peri-cholecystic fluid & Present & $8.5(0.95-76.94)$ & 0.0559 \\
\hline AST & $>50 \mathrm{U} / \mathrm{L}$ & $0.247(0.1-0.59)$ & 0.0018 \\
\hline ALT & $>43 \mathrm{U} / \mathrm{L}$ & $0.318(0.13-0.76)$ & 0.0097 \\
\hline Alkaline phosphatase & $>200 \mathrm{U} / \mathrm{L} 0.147$ & $(0.03-0.69)$ & 0.0148 \\
\hline
\end{tabular}


Fro patients with $\mathrm{AC}$, the mean operation time was longer $(\mathrm{P}<.001)$ and the mean blood loss during LC was less $(\mathrm{P}<.001)$.

\section{Discussion:}

$\mathrm{AC}$ is associated with a higher grade of operative difficulty and postoperative morbidity. Therefore, considering the many adverse influences on the patients and the postoperative outcomes, it is desirable to select the subgroup of patients who are more prone to develop AC.7,8 Furthermore, for the patients at high risk of developing $\mathrm{AC}$, it is better to operate before AC develops. ${ }^{8}$ In the present study, multiple factors are considered high risk for $\mathrm{AC}$ on multivariate analysis. These factors include comorbidities, such as diabetes, 2 demographic factors, including age older than 51 years and male sex. Presence of leukocytosis, peri-cholecystic collection, and elevated liver enzymes are high predictor factors for AC.

Also, It has been reported that the prevalence of the anomalous cystic duct confluence and accessory hepatic duct are $3.7-18$ and $2.9-27 \%$, respectively. ${ }^{9}$ In this series of 96 cases, the incidences are 0.6 and $2.0 \%$, respectively.

MRCP is the most useful modality when the cystic duct is occluded or bile and pancreatic bi-ductal imaging is needed to diagnose pancreaticobiliary malunion. ${ }^{10}$ Nevertheless, there are still undoubtedly some anomalies that could be missed by all available diagnostic modalities. ${ }^{11}$ Many authors have reported that routine IOC reduces risk and severity of intraoperative common bile duct injury and helps identify the site of injury. ${ }^{11,12}$ However, injuries to accessory hepatic ducts or anomalous cystic ducts cannot be prevented by IOC and in many cases of acute complicated or cholecystitis it is difficult to proceed for IOC or even to cannulate the cystic duct which is rarely identified in these cases. Therefore, careful dissection of the cystic duct and artery at Calot's triangle is of utmost importance in the recognition of bile duct anomalies which may necessitate proper identification of bile duct anatomy. ${ }^{13}$
This study has shown some benefits of the preoperative placement of endonasal biliary tube drainage (ENBTD) to prevent intraoperative bile duct injury in cases with probable bile duct anomalies. There have been reports advocating preoperative placement of ENTD tubes or endoscopic nasogallbladder drainage (ENGBD) tubes for the same purpose in cases of acute cholecystitis complicated by severe inflammation or bile duct anomalies. ${ }^{14}$ Recently, some endoscopists have revealed that ENBTD is feasible and safe. Despite established biliary drainage using a conventional duodenoscope, one of greatest motivations to perform the ENBTD may be that it is minimally invasive even for critically ill patients. ${ }^{14,15}$ The merits of ENBTD assisted LC as concluded from this study are as follows:

1. ENBTD has the advantage of not only serving endoscopic drainage of the biliary tree but delineating the biliary tree via cholangiography and detecting biliary tract anomalies especially in patients with difficulties in identifying the anatomy as the studied group (acute cholecystititis).

2. IOC can be repeated intraoperatively using the ENBTD tube if necessary, especially if the surgeon was unable to visualize the cystic duct exterior because of severe inflammation, adhesions around the Calot's triangle, or biliary tract anomalies.

3. It helped to distinguish the anomalous hepatic duct from the cystic duct and aberrant cystic duct by sensing presence of the ENBTD tube during LC and this is stated by other studies. 16,17 Kuroki and his associates similarly reported the usefulness of this technique to identify the anatomy within the hepato-duodenal ligament. ${ }^{18}$

4. It decreased the rate of conversion to laparotomy in this study to zero even with presence of both acute inflammation and anomalies in the anatomy.

5. The cystic duct would be able to be distinguished from the accessory hepatic duct accurately by using the ENBD tube.

ERCP and subsequent ENBTD tube placement are well known risk factors 
for serious complications such as acute pancreatitis, sepsis, and bleeding. ${ }^{18}$ In this series, we experienced a single case of post-intervention pancreatitis. Rates of ERCP-related morbidity and mortality have been reported to be $1-7$ and $0.2-1 \%$, respectively. Uchiyama et al. recommended the preoperative placement of ENBTD tubes except in cases of anomalous cystic duct and accessory hepatic duct type, as the bile duct injuries in his series occurred in cases of anomaly types. ${ }^{19}$ After weighing the contribution of ENBTD tube placement to decreased intraoperative bile duct injury relative to its association with post ERCP pancreatitis, ENBD tube placement prior to LC may have successfully decreased the incidence of complications during $\mathrm{LC}$ for acute or complicated cholecystitis.

In conclusion, male sex, age older than 51 years and diabetes increase the probability of developing $\mathrm{AC}$ in patients with symptomatic gallstones, and in suspected cases with acute or complicated cholecystitis ENBTD can be used safely to identify the bile duct and detect anomalies so we can avoid bile duct injuries in these cases.

\section{References:}

1- Itoi T, Tani M, Kawai M, Ueno M, Hama $\mathrm{T}$, Yamaue $\mathrm{H}$ : Preoperative evaluation of the extrahepatic bile duct structure for laparoscopic cholecystectomy. Surg Endosc 2006; 20: 1119-1123.

2- Adamsen S, Hansen OH, FunchJensen P, Schulze S, Stage JG, Wara $\mathrm{P}$ : Bile duct injury during laparoscopic cholecystectomy: A prospective nationwide series. J Am Coll Surg 1997; 184: 571-578.

3- Tantia O, Jain M, Khanna S, Sen B: Iatrogenic biliary injury: 13,305 cholecystectomies experienced by a single surgical team over more than 13 years. Surg Endosc 2008; 22: 1077-1086.

4- MacFadyen BV Jr, Vecchio R, Ricardo AE, Mathis CR: Bile duct injury after laparoscopic cholecystectomy. The United States experience. Surg Endosc1998; 12: 315-321.
5- Braghetto I, Bastias J, Csendes A, Debandi A. Intraperitoneal bile collections after laparoscopic cholecystectomy: Causes, clinical presentation, diagnosis, and treatment. Surg Endosc 2000; 14: 1037-1041.

6- Ko K, Kamiya J, Nagino M, Oda K, Yuasa $\mathrm{N}$, Arai T, et al: A study of the subvesical bile duct (duct of Luschka) in resected liver specimens. World J Surg 2006; 30: 1316-1320.

7- Kurumi Y, Tani T, Hanasawa K, Kodama M: The prevention of bile duct injury during laparoscopic cholecystectomy from the point of view of anatomic variation. Surg Laparosc Endosc Percutan Tech 2000; 10: 192-199.

8- Liyanage CA, Sadakari Y, Kitada H, Ienaga J, Tanabe R, Takahata S, et al: Prevention of iatrogenic bile duct injuries in difficult laparoscopic cholecystectomies: Is the naso-biliary drain the answer? $J$ Hepatobiliary Pancreat Surg 2009; 16: 458-462.

9- Kuroki T, Tajima Y, Tsuneoka N, Kitasato A, Adachi T, Mishima T, et al: Biliary navigation surgery using endoscopic nasobiliary drainage tube for the prevention of bile duct injury in laparoscopic cholecystectomy. Hepatogastroenterology 2007; 54: 1018-1019.

10-Toyota N, Takada T, Amano H, Yoshida M, Miura F, Wada K: Endoscopic nasogallbladder drainage in the treatment of acute cholecystitis: Alleviates inflammation and fixes operator's aim during early laparoscopic cholecystectomy. $J$ Hepatobiliary Pancreat Surg 2006; 13: 80-85.

11-Mergener K, Strobel JC, Suhocki P, Jowell PS, Enns RA, Branch MS, et al: The role of ERCP in diagnosis and management of accessory bile duct leaks after cholecystectomy. Gastrointest Endosc 1999; 50: 527-531.

12-Shaw MJ, Dorsher PJ, Vennes JA: Cystic duct anatomy: An endoscopic perspective. Am J Gastroenterol 1993; 88: 2102-2106. 13-Debru E, Dawson A, Leibman S, 
Richardson M, Glen L, Hollinshead $\mathrm{J}$, et al: Does routine intraoperative cholangiography prevent bile duct transection? Surg Endosc 2005; 19: 589-593.

14-Kullman E, Borch K, Lindstrom E, Svanvik J, Anderberg B: Value of routine intraoperative cholangiography in detecting aberrant bile ducts and bile duct injuries during laparoscopic cholecystectomy. Br J Surg 1996; 83: 171-175.

15-Spanos CP, Syrakos T: Bile leaks from the duct of Luschka (subvesical duct): A review. Langenbecks Arch Surg 2006; 391: 441-447.

16-Wong YC, Wang LJ, Chen RJ, Chen CJ: Magnetic resonance imaging of extrahepatic bile duct disruption. Eur Radiol 2002; 12: 2488-2490.

17-Kuroki, Ausch C, Hochwarter G, Taher
M, Holzer B, Rosen HR, Urban M, et al: Improving the safety of laparoscopic cholecystectomy: The routine use of preoperative magnetic resonance cholangiography and ENBTD. Surg Endosc 2005; 19: 574-580.

18-Hirao K, Miyazaki A, Fujimoto T, Isomoto I, Hayashi $\mathrm{K}$ : Evaluation of aberrant bile ducts before laparoscopic cholecystectomy: Helical CT cholangiography versus MR cholangiography. AJR Am J Roentgenol. 2000; 175: 713-720.

19-Uchiyama K, Kawai T, Sofuni A, Itokawa F, Tsuchiya T, Kurihara T, Kusano C, Saito Y, Gotoda T: Efficacy and safety of 1-step transnasal endoscopic nasobiliary drainage for the treatment of acute cholangitis in patients with previous endoscopic sphincterotomy (with videos). Gastrointest Endosc 2008; 68: 84-90. 